\title{
Aberration Measurement of the Probe-Forming System of an Electron Microscope using Two-Dimensional Materials
}

\author{
Hidetaka Sawada ${ }^{1,2,3,4^{*}}$ Christopher S. Allen ${ }^{1,2}$, Shanshan Wang ${ }^{2}$, Jamie H. Warner ${ }^{2}$, and Angus I. Kirkland ${ }^{1,2}$ \\ ${ }^{1}$ Electron Physical Sciences Imaging Centre (ePSIC), Diamond Light Source Ltd, Didcot, Oxford, OX11 0DE, \\ UK \\ ${ }^{2}$ Department of Materials, University of Oxford, Parks Road, Oxford OX1 3PH, UK \\ ${ }^{3}$ JEOL UK Ltd., Silver Court Watchmead Welwyn Garden City, Herts., AL7 1LT, UK \\ ${ }^{4}$ Research acceleration program, Japan Science and Technology Agency, K's Gobancho, 7, Gobancho,
} Chiyoda-ku, Tokyo, 102-0076 Japan

*E-mail: hide.sawada@materials.ox.ac.uk

[Key words] Geometric Aberration, Chromatic Aberration, STEM, Ronchigram, Aberration Corrector

\section{PACS: 07.05.Kf; 68.37.Ma}

\begin{abstract}
The geometric and chromatic aberration coefficients of the probe-forming system in an aberration corrected transmission electron microscope have been measured using a Ronchigram recorded from monolayer graphene. The geometric deformations within individual local angular sub-regions of the Ronchigram were analyzed using an auto-correlation function and the aberration coefficients for the probe forming lens were calculated. This approach only requires the acquisition of a single Ronchigram allowing rapid measurement of the aberration coefficients. Moreover, the measurement precision for defocus and two-fold astigmatism is improved over that which can be achieved from analysis of Ronchigrams recorded from amorphous films. This technique can also be applied to aberration corrected STEM imaging of any hexagonal two-dimensional material.
\end{abstract}

\section{Introduction}

Aberration correctors in a modern Scanning Transmission Electron Microscopes (STEM) consist of combinations of multipole elements, deflectors and in some optical configurations round lenses [1-4]. The relationships between the strength of the various optical elements in the corrector and the aberrations in the STEM probe are complex and interconnected and in order to optimize the size and shape of the STEM probe for imaging and analysis [5] an accurate automated alignment system is required to measure and compensate residual aberrations in the STEM probe-forming optics.

A common method for measurement of the aberrations in a STEM probe involves measurement of deformations in pairs of over and underfocus images of nm sized metal particles supported on a carbon film [6]. Alternatively, the Ronchigram can be used to calculate the aberrations from suitably conditioned measurements of beam induced image shifts [7], contrast transfer functions [8], segmentation of Ronchigrams of amorphous films [9] or a crystalline lattice [10-13]. Finally, datasets used for electron ptychography can also provide direct measurements of probe forming aberrations [14].

As previously reported, auto-correlation functions calculated from defined areas of the Ronchigram recorded from a thin amorphous film [9] can be used to analyze the second-order derivative of the wave aberration 
function from which aberrations for the probe-forming system can be calculated. Previous reports [11-13] have also described methods for aberration measurement using local Ronchigrams calculated from a crystalline sample from measurements of the magnifications in local angular areas. This latter method is both fast and accurate since only a single Ronchigram is required and the local magnification is readily determined from high contrast features arising from the periodic lattice.

However, although the computational analysis of a single Ronchigram of a crystalline sample is fast it requires orientation of a sample to a specific, known zone axis which takes time and requires significant manual skill. A more fundamental problem with these methods is that a finite, generally unknown specimen thickness leads to inaccuracies in defocus measurement.

In this work auto-correlation patterns calculated from segmented areas of a Ronchigram of monolayer graphene have been used to determine the local magnification and hence the aberrations in the probe forming system. The use of a thin two-dimensional material, in this case graphene, overcomes both the practical difficulties of zone axis alignment and the fundamental limitations associated with thick specimens. Furthermore, the features in an angular area of a two-dimensional lattice are robust to beam or specimen tilt compared to those in thicker crystalline specimens as the reciprocal lattice of a thin two-dimensional structure is extended along the electron trajectory in the $z$ direction. For practical implementation we note that high quality graphene TEM samples are now commercially available [14].

\section{Theory}

A convergent beam impinging on a sample plane produces a Ronchigram in the far field [16]. This Ronchigram can be considered as the pattern formed from an electron probe focused at or near the specimen plane, projected to a far plane (determined by the camera length of the projector optics). When the probe-forming system contains geometrical aberrations, the electron beam does not converge to a single crossover, but instead, the crossover position depends on local angular position, as shown schematically in Figure 1(a). As a consequence, axial aberrations in the probe-forming lens system are visible in the Ronchigram $[17,18]$.

Figures 1(b-d) show calculated Ronchigrams of a geometric hexagonal lattice in the presence of various low order geometrical aberrations. In all cases, the hexagonal symmetry of the simulated Ronchigram is distorted by the presence of geometric aberrations in the probe-forming lens.

The lattice spacing within a local angular area of a Ronchigram, D, is described by $[7,18]$;

$$
D=\frac{L \cdot d}{z}=\frac{L \cdot d}{\left(\frac{\partial G_{\alpha}}{\partial \alpha}\right)}=M \cdot d .
$$

where $L$ is the camera length, $d$ the lattice spacing of the specimen, $z$ the distance between the probe crossover and the specimen plane and with $M$ the local magnification. For an aberrated beam, $z$ is defined by the differential of the geometrical aberration $\left(G_{\alpha}\right)$ with respect to the convergence semi-angle $(\alpha)$. 
Equation (1) shows that the value of the local magnification varies across the Ronchigram, leading to an overall distortion described by the product of $L$ and the differential of the geometric aberration, $M=L \cdot\left(\frac{\partial G_{\alpha}}{\partial \alpha}\right)^{-1}[7,18]$.

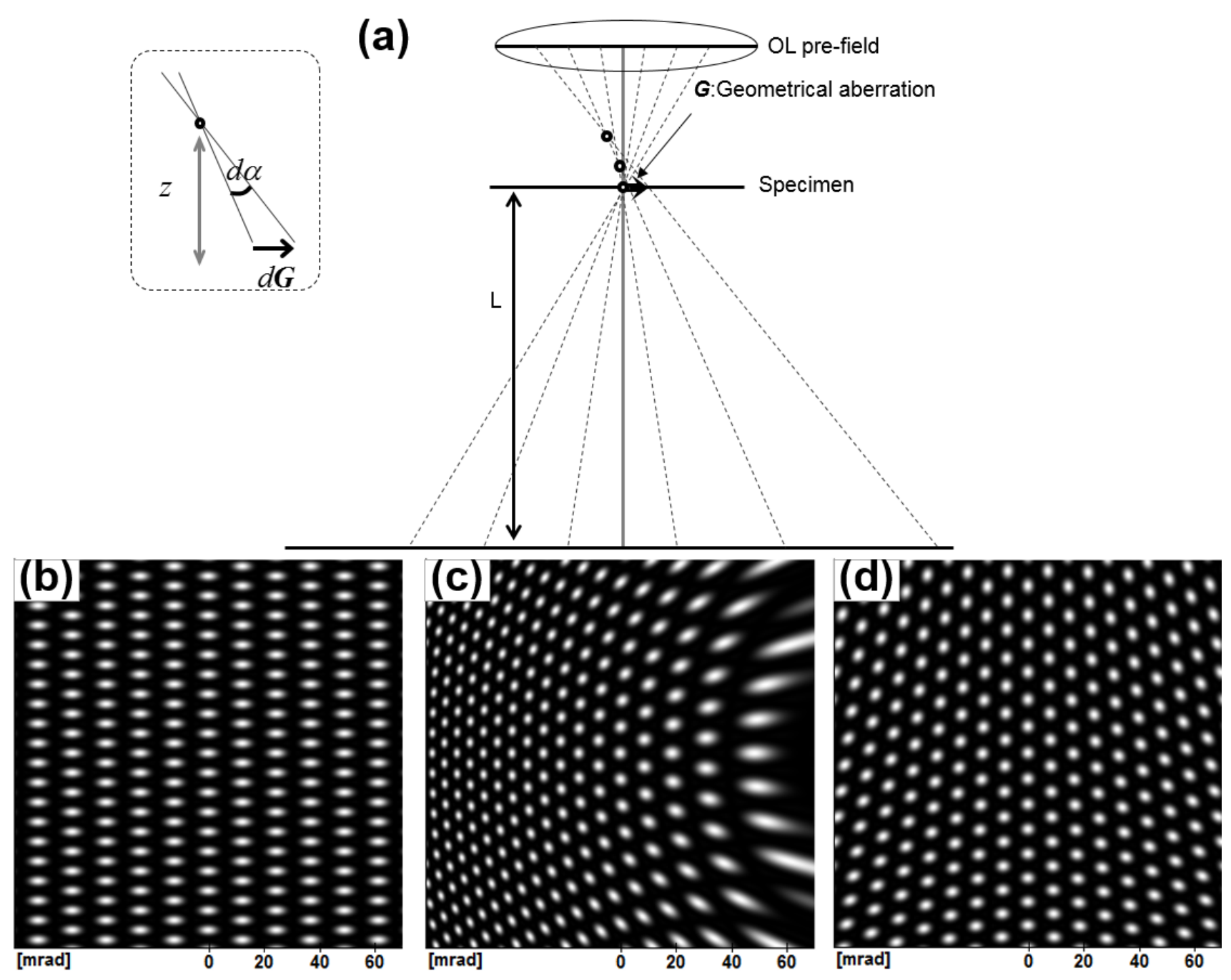

Figure 1. (a) Schematic ray diagram for the formation of a Ronchigram in the presence of geometric aberrations. Calculated Ronchigrams with (b) two-fold astigmatism, $a 2=10 \mathrm{~nm}$ and $\theta_{a 2}=0^{\circ}$, (c) axial coma, $p 3=200 \mathrm{~nm}$ and $\theta_{p 3}$ $=0^{\circ}$ and (d) three-fold astigmatism, $a 3=30 \mathrm{~nm}$ and $\theta_{a 3}=30^{\circ}$. Defocus, $o 2=-30 \mathrm{~nm}$ in (b)-(d). The simulated specimen in all cases is a simple hexagonal lattice with a lattice constant of $0.246 \mathrm{~nm}$.

Distortions in a segment of a Ronchigram (Figure 2) are characterized by the geometric aberration function (Table A1) as;

$$
\left.\mathbf{G}\left(\boldsymbol{\omega}_{0}+\Delta \omega\right) \approx \Delta \mathbf{G}\right|_{\omega 0} \cdot \Delta \omega=\left.\left(\Delta G_{u}, \Delta G_{v}\right)\right|_{\omega 0}
$$

where

$$
\begin{gathered}
\left.\Delta G_{u}\right|_{\omega 0}=A \cdot \Delta \omega_{u}+B \cdot \Delta \omega_{v} \\
\left.\Delta G_{v}\right|_{\omega 0}=B \cdot \Delta \omega_{u}+C \cdot \Delta \omega_{v}
\end{gathered}
$$


and

$$
A=\left.\frac{\partial G_{u}}{\partial \omega_{u}}\right|_{\omega \theta}, \quad B=\left.\frac{\partial G_{u}}{\partial \omega_{v}}\right|_{\omega \theta}, \quad C=\left.\frac{\partial G_{v}}{\partial \omega_{v}}\right|_{\omega \theta}
$$

In equation (2) $\boldsymbol{\omega}_{\mathbf{0}}$ is a vector defining the position of the centre of a segment in the Ronchigram with respect to the pattern centre and where $\left(\Delta \omega_{u}, \Delta \omega_{v}\right)$ are small distances along directions $(u, v)$, that define the reciprocal space co-ordinates the Ronchigram [4].

The factors $A, B$, and $C$ in equation (5) and are listed in Table $\mathrm{A} 2$ for all aberration coefficients up to fifth order.

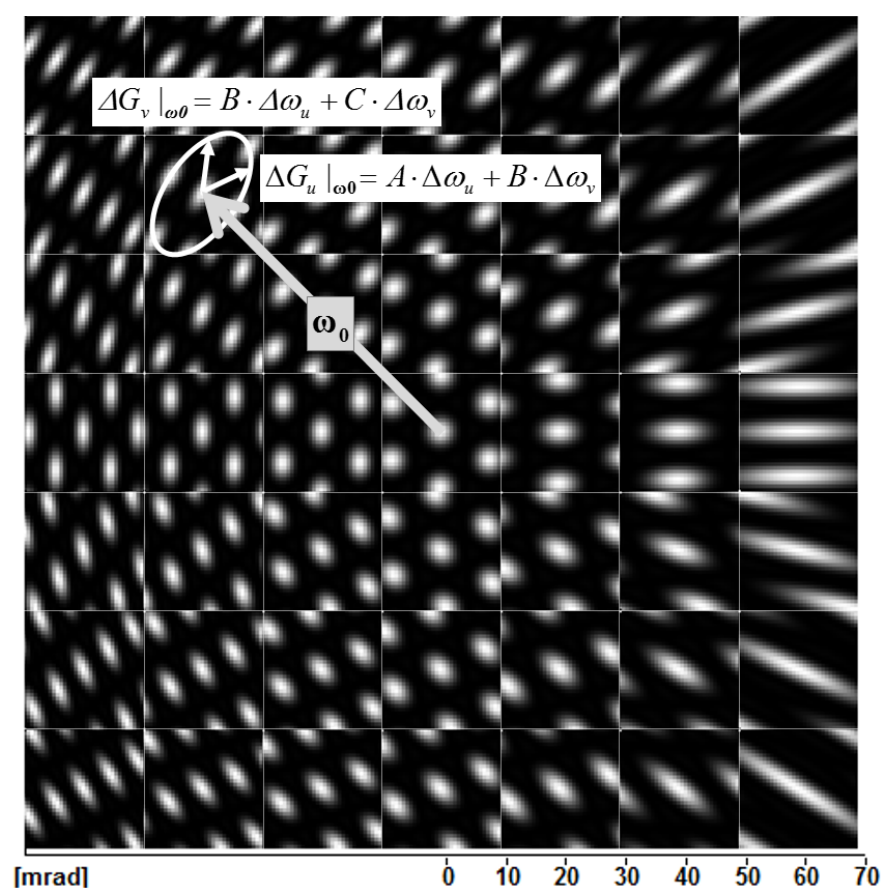

Figure 2. Simulated segmented Ronchigram illustrating the definition of local geometric aberrations. Each of the $7 \mathrm{x}$ 7 segments in the simulation were separately calculated using equation (2) with values of $P 3=200 \mathrm{~nm}\left(\theta_{P 3}=0^{\circ}\right)$ and $O 2=-30 \mathrm{~nm}$, corresponding to the aberrations used to calculate Figure 1(c).

\section{Experimental}

Aberration coefficients were measured from data recorded using a JEM-ARM300F equipped with STEM and TEM ETA spherical aberration correctors [3]. Ronchigrams were recorded at an accelerating voltage of $80 \mathrm{kV}$ from a monolayer graphene sample on a Gatan Oneview camera with 4096 x 4096 pixels using a focused STEM probe (Figure 3(a)). The Ronchigram was divided into local angular areas and the lateral magnification change in two directions for each segment was measured using the auto-correlation function (Figure 3(b)). As a consequence of symmetry the auto-correlation function from each segment consists of six secondary peaks in a mirror symmetric arrangement surrounding the central peak and hence only half the peaks (marked I, II, and III in Figure 3(b)) were 
used for fitting. The positions of the maxima of these three peaks used were located to sub-pixel accuracy with parabolic fits and the resultant peak positions fitted to an oval function given by;

$$
\left(A \cdot \Delta \omega_{u}+B \cdot \Delta \omega_{v}\right)^{2}+\left(B \cdot \Delta \omega_{u}+C \cdot \Delta \omega_{v}\right)^{2}=1
$$

Subsequently, from these fitted parameters the aberrations for the probe forming lens were calculated from;

$$
\left.\Delta G_{u}\right|_{\omega 0}{ }^{2}+\left.\Delta G_{v}\right|_{\omega 0}{ }^{2}=d^{2} \cdot L^{2} \cdot\left\{\left(A \cdot \Delta \omega_{u}+B \cdot \Delta \omega_{v}\right)^{2}+\left(B \cdot \Delta \omega_{u}+C \cdot \Delta \omega_{v}\right)^{2}\right\}
$$

where $d$ is the lattice parameter of graphene and $L$ is the camera length. Since the lattice parameter of graphene is known only one Ronchigram is required to calculate the aberration coefficients.

In pixel coordinates equation 7 can be re-written as;

$$
\left.\Delta G_{u}\right|_{\omega 0}{ }^{2}+\left.\Delta G_{v}\right|_{\omega 0}{ }^{2}=d^{2} / k^{2} \cdot\left\{\left(A_{[1 / p i x]} \cdot \Delta \omega_{u}+B_{[1 / p i x]} \cdot \Delta \omega_{v}\right)^{2}+\left(B_{[1 / p i x]} \cdot \Delta \omega_{u}+C_{[1 / p i x]} \cdot \Delta \omega_{v}\right)^{2}\right\}
$$

where $k[\mathrm{rad} / \mathrm{pixel}]$ is the angular calibration factor calibrated using graphene reflections as shown in Figure 3(c).
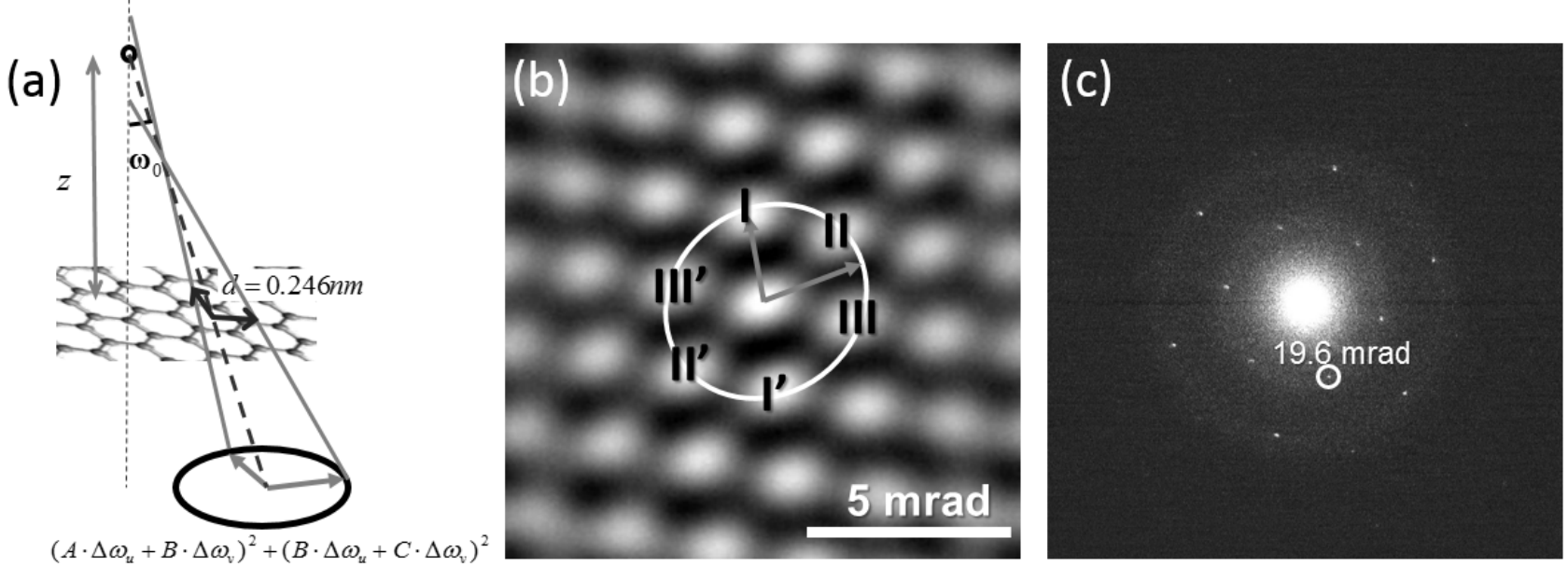

Figure 3. (a) Schematic diagram showing the relationship between a graphene specimen and the corresponding oval shape in the Ronchigram. (b) Auto-correlation pattern calculated from a segmented area of an experimental Ronchigram calculated from a monolayer graphene specimen showing peak positions (with those generated from mirror symmetry marked with a prime) and the oval fit to three peaks. (c) Diffraction pattern recorded from a monolayer graphene sample using parallel illumination. 


\section{Results}

\subsection{Defocus measurement.}

Figures 4(a-j) show experimental Ronchigrams recorded at $80 \mathrm{kV}$ with a camera length of $40 \mathrm{~cm}$ from a monolayer graphene sample [15] with a $2 \mathrm{X}$ pixel binning for linear objective lens focus changes in 10 bit increments. For this data an area of $700 \times 700$ pixels was extracted with 1 pixel corresponding to an angular range of $66.18 \mu$ rad. The Ronchigram was then segmented into $3 \times 3$ local angular areas $(256 \times 256$ pixels $)$ and auto-correlation patterns calculated from each area. The auto-correlation pattern from the central local area is shown at the lower left of each Ronchigram (Figure 4) and the result of the oval fit to equation 6 showing a gradual change in size with defocus is indicated by the red line. The value of defocus measured from each Ronchigram is shown in Figure $4(\mathrm{k})$. These measured values were fitted to a linear function with a slope of $0.75 \mathrm{~nm} / \mathrm{bit}$ corresponding to the defocus increment / bit of the objective lens at $80 \mathrm{kV}$. In Figure 4here is a small residual two fold astigmatism giving rise to the small oval distortion visible in $(\mathrm{h}-\mathrm{j})$.
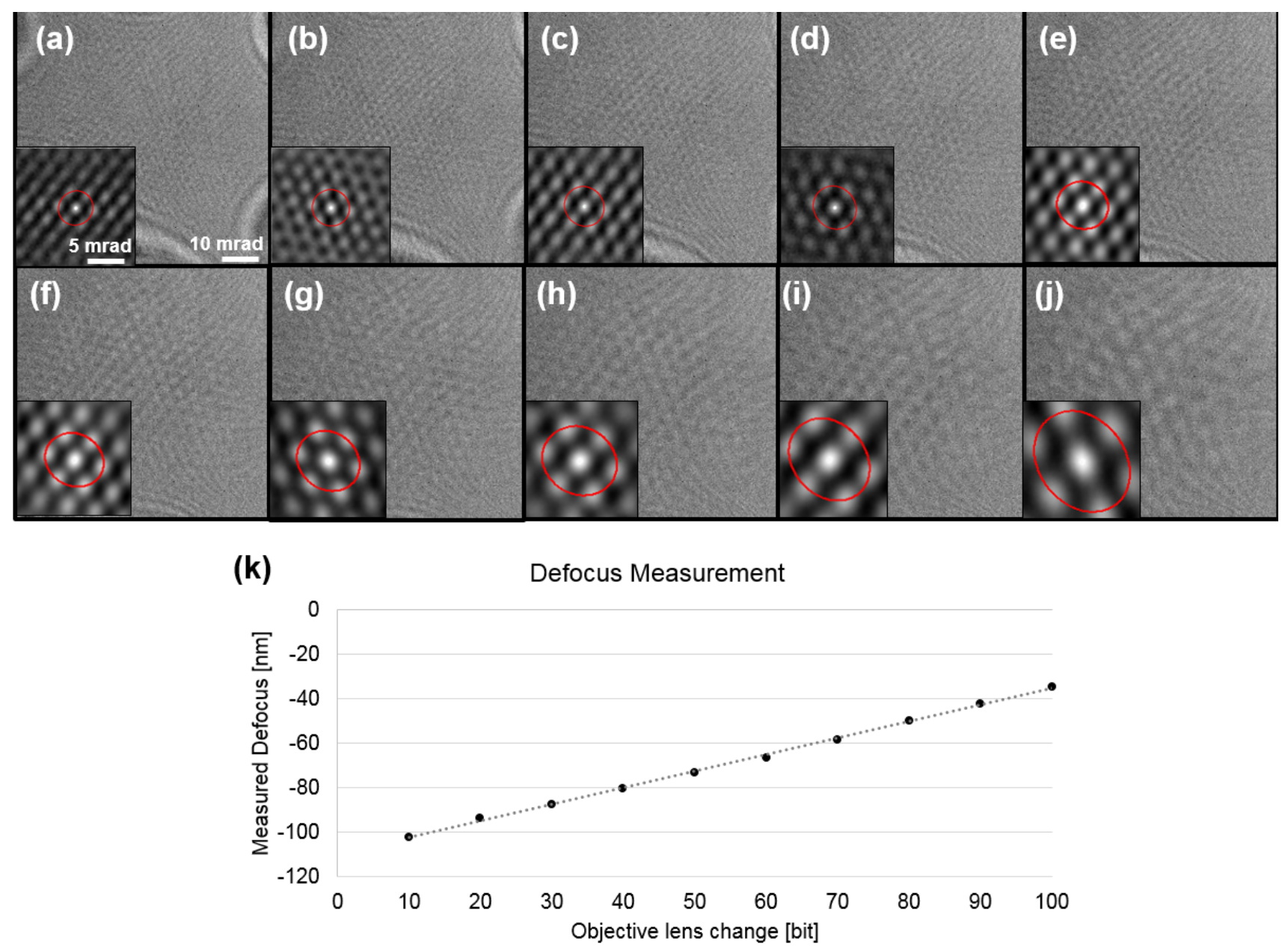

Figure 4. (a-j) Experimental Ronchigrams (700 $\times 700$ pixels) recorded from monolayer graphene for known changes in objective lens excitation (10 bit increments). Insets show enlargements $(256 \times 256$ pixels $)$ of the central angular region of the full auto-correlation pattern. (k) Measured defocus calculated from (a-j) as a function of change in the objective lens excitation together with a least squares fitted linear function with a slope of 0.75 . 


\subsection{Measurement of axial-coma and three-fold astigmatism.}

Figure 5(a) shows a Ronchigram recorded at $80 \mathrm{kV}$ with a camera length of $40 \mathrm{~cm}$ with a $2 \mathrm{X}$ pixel binning from monolayer graphene [15] with a focused STEM probe in which a known $2^{\text {nd }}$ order axial coma was intentionally introduced. For this data an area of $650 \times 650$ pixels was extracted with 1 pixel corresponding to an angular range of $66.18 \mu \mathrm{rad}$. The Ronchigram was then segmented into $3 \times 3$ local angular areas $(256 \times 256$ pixels $)$ and autocorrelation patterns calculated from each area. Fits to the six peaks in the auto-correlation pattern for each segment are shown in Figure 5(b). The size of the fitted oval functions from segments extracted from the right hand side of the Ronchigram are larger than those from the left and the shape of the fitted function also changes from right to left. These effects are due to the local defocus at the right of the Ronchigram being closer to the Gaussian focus as a result of the introduced $2^{\text {nd }}$ order axial-coma. Table 1 lists the aberration coefficients up to third order calculated from the oval fits shown in Figure 5(b) including a large value of $2^{\text {nd }}$ order axial coma value closely corresponding to that introduced.

Similarly Figure 5(d) shows a Ronchigram recorded from monolayer graphene under the same conditions as in Figure 5(a) but for which a known value of three-fold astigmatism has been deliberately introduced together with corresponding fits to the auto-correlation pattern for each segment (Figure 5(e)). A three-fold symmetry is evident in the orientation of the 9 fitted oval functions. Table 2 lists the aberration coefficients up to third order calculated from the oval fits shown in Figure 5(d) including a large value for three-fold astigmatism corresponding closely to that deliberately introduced. Figures 5(c) and (f) show simulated Ronchigrams using the measured values of the aberrations in Tables 1 and 2 respectively. The magnification change and deformation in the simulated Ronchigrams reproduces the experimental data in Figures 5 (a) and 5(d), indicating that the analysis described correctly determines these geometric aberrations in the probe-forming system to high accuracy. 
(a)

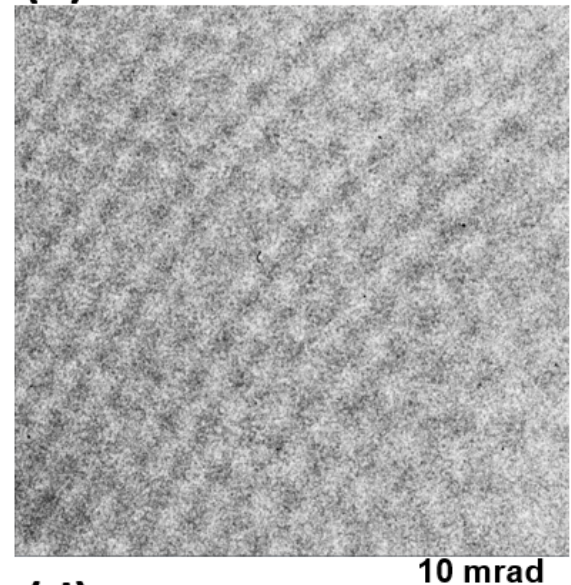

(d)

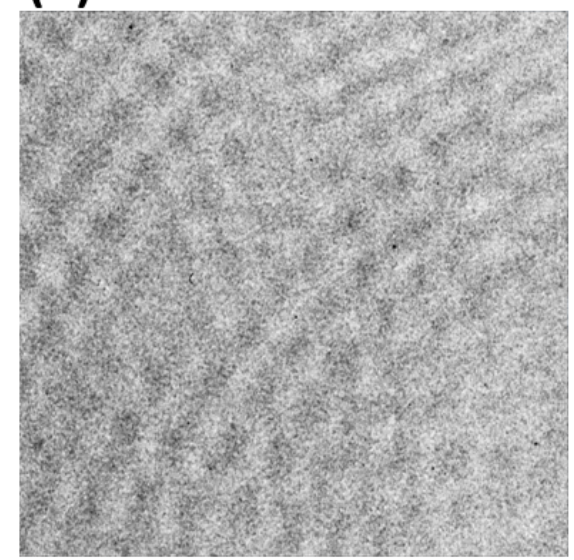

$10 \mathrm{mrad}$ (b)

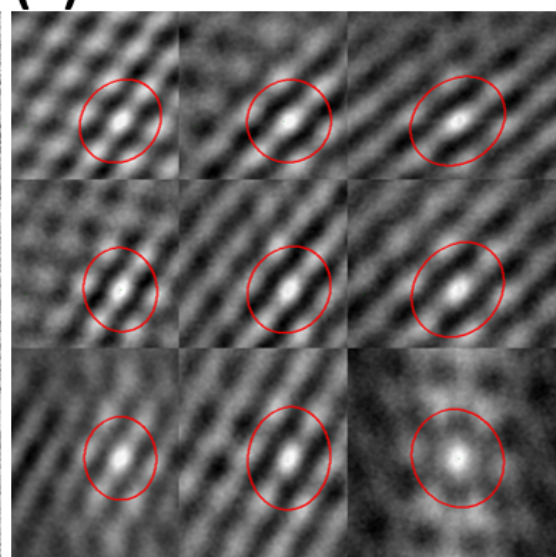

(e)

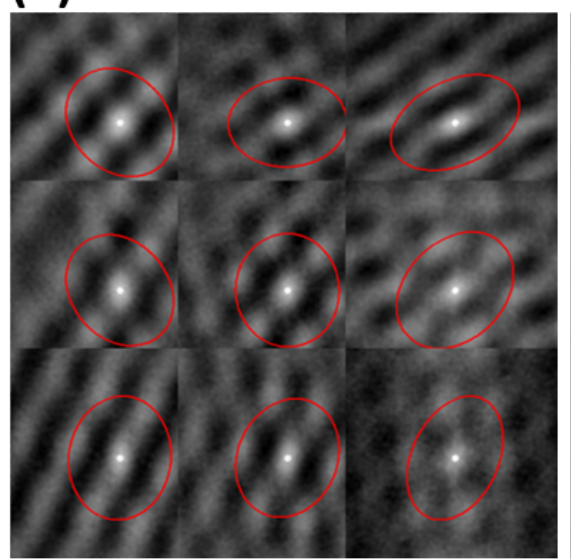

(c)

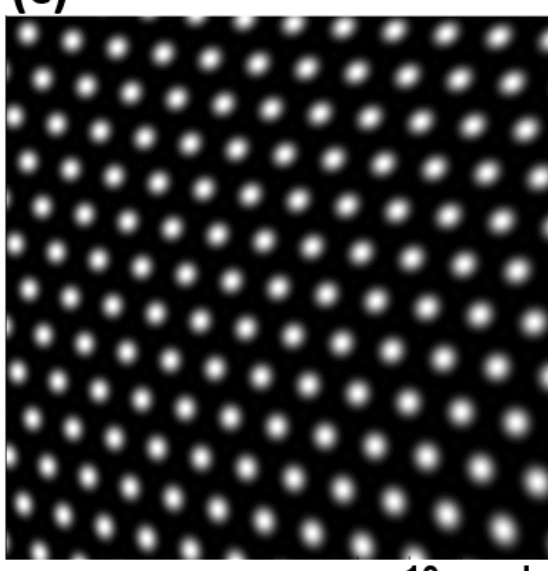

$10 \mathrm{mrad}$

(f)

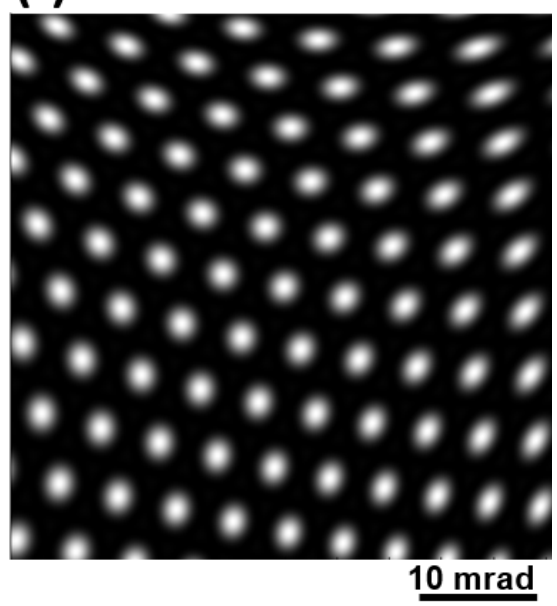

Figure 5. (a) and (d) Experimental Ronchigrams $(650 \times 650$ pixels $)$ recorded from monolayer graphene with (a) $2^{\text {nd }}$ order axial coma (of order 100nm) and (d) three-fold astigmatism (of order 100nm) intentionally introduced. (b) and (e) Local area auto-correlation functions $(650 \times 650$ pixels $)$ calculated from the Ronchigrams in (a) and (d) together with corresponding oval fits. (c) and (f) Simulated Ronchigrams for a simple hexagonal lattice with $d=0.246 \mathrm{~nm}$ using the aberration coefficients measured from (b) and (e) respectively (Tables 1 and 2). 
Table 1. Measured aberration coefficients to third order of the probe forming system calculated from the measurements shown in Figures 5(a and b). Values in brackets correspond to errors in the measurement values. The error in azimuth is described by $360^{\circ}$ /symmetry expressed as a percentage.

\begin{tabular}{|l|l|l|}
\hline Aberration & Amplitude & Azimuth \\
\hline O2: Defocus & $-74.35[\mathrm{~nm}](0.36[\mathrm{~nm}])$ & \\
\hline A2: Two-fold stigmatism & $4.01[\mathrm{~nm}](1.00[\mathrm{~nm}])$ & $60.03^{\circ}(2.49 \%)$ \\
\hline P3: Axial Coma & $330.64[\mathrm{~nm}](15.54[\mathrm{~nm}])$ & $-13.83^{\circ}(1.90 \%)$ \\
\hline A3: Three-fold astigmatism & $72.16[\mathrm{~nm}](19.00[\mathrm{~nm}])$ & $35.30^{\circ}(4.81 \%)$ \\
\hline A4: Four-fold astigmatism & $0.44[\mu \mathrm{m}](0.75[\mu \mathrm{m}])$ & $-43.25^{\circ}(32.59 \%)$ \\
\hline Q4: Star aberration & $3.98[\mu \mathrm{m}](1.42[\mu \mathrm{m}])$ & $-59.80^{\circ}(7.00 \%)$ \\
\hline O4: $3^{\text {rd }}$ order spherical aberration & $0.98[\mu \mathrm{m}](0.80[\mu \mathrm{m}])$ & \\
\hline
\end{tabular}

Table 2. Measured aberration coefficients to third order of the probe forming system calculated from the measurements shown in Figures 5(d and e). Values in brackets correspond to errors in measurement values. The error in azimuth is described by $360^{\circ} /$ symmetry expressed as a percentage.

\begin{tabular}{|l|l|l|}
\hline Aberration & Amplitude & Azimuth \\
\hline O2: Defocus & $-58.21[\mathrm{~nm}](0.77[\mathrm{~nm}])$ & \\
\hline A2: Two-fold stigmatism & $2.08[\mathrm{~nm}](0.74[\mathrm{~nm}])$ & $59.66^{\circ}(6.80 \%)$ \\
\hline P3: Axial Coma & $53.23[\mathrm{~nm}](24.24[\mathrm{~nm}])$ & $-116.79^{\circ}(7.52 \%)$ \\
\hline A3: Three-fold astigmatism & $243.77[\mathrm{~nm}](14.07[\mathrm{~nm}])$ & $25.89^{\circ}(1.13 \%)$ \\
\hline A4: Four-fold astigmatism & $3.21[\mu \mathrm{m}](0.70[\mu \mathrm{m}])$ & $-54.46^{\circ}(4.45 \%)$ \\
\hline Q4: Star aberration & $4.15[\mu \mathrm{m}](2.32[\mu \mathrm{m}])$ & $18.66^{\circ}(5.61 \%)$ \\
\hline O4: $3^{\text {rd }}$ order spherical aberration & $-1.28[\mu \mathrm{m}](1.10[\mu \mathrm{m}])$ & \\
\hline
\end{tabular}

\subsection{Aberration measurement to $5^{\text {th }}$ order}

By increasing the number of auto-correlation patterns measured, higher order aberrations can also be measured using the method described. Figure 6(a) shows a Ronchigram recorded at $80 \mathrm{kV}$ with a camera length of $80 \mathrm{~cm}$ from a monolayer graphene sample [15] with a $1 \mathrm{X}$ pixel binning corresponding to an angular range up to 32 mrad with1 pixel corresponding to $16.04 \mu \mathrm{rad}$. Aberration coefficients up to $5^{\text {th }}$ order were measured using an array of 37 segmented areas each of $600 \times 600$ pixels (Figure 6(b)). Values of the measured aberration coefficients up to $5^{\text {th }}$ order are listed in Table 3. Figure 6(c) shows a high-resolution ADF STEM image of a monolayer graphene sample recorded with the aberration conditions listed in Table 3 for which a small additional adjustment of defocus and two-fold astigmatism was performed after inserting the condenser aperture. 

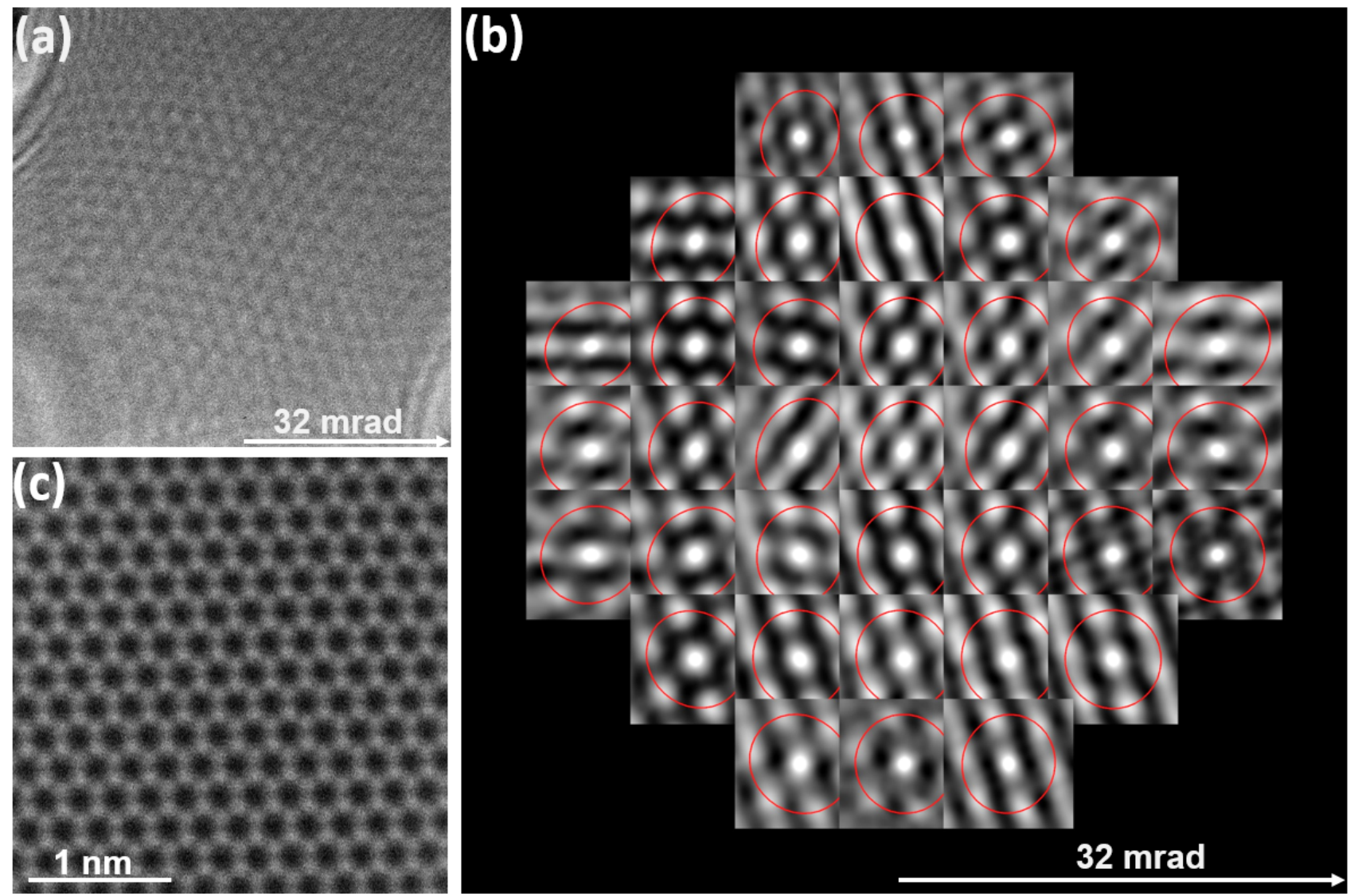

Figure 6. (a) Experimental Ronchigram recorded from monolayer graphene. (b) Local area auto-correlation pattern from an array of 37 sub-areas $(600 \times 600$ pixels $)$ and fitted oval functions. (c) Low angle annular dark field (LAADF) image of monolayer graphene at $80 \mathrm{kV}$ recorded with a convergence semi-angle of $25.2 \mathrm{mrad}$ and beam current of $23 \mathrm{pA}$ using the aberration conditions listed in Table 3. The inner and outer detection angles for the LAADF image are $39 \mathrm{mrad}$ and $180 \mathrm{mrad}$ respectively. Ten frames were averaged to produce the image in (c) with a dwell time of $100 \mu \mathrm{s} /$ pixel with $8.2 \mathrm{pm} / \mathrm{pixel}$ for each frame. 
Table 3: Measured aberration coefficients to fifth order of the probe forming system calculated from the measurements shown in Figure 6. Values in brackets correspond to errors in measurement values. The error in azimuth is described by $360^{\circ} /$ symmetry expressed as a percentage.

\begin{tabular}{|l|l|l|}
\hline Aberration & Amplitude & Azimuth \\
\hline O2: Defocus & $-71.46[\mathrm{~nm}](0.30[\mathrm{~nm}])$ & \\
\hline A2: Two-fold stigmatism & $3.090[\mathrm{~nm}](0.31[\mathrm{~nm}])$ & $73.44^{\circ}(1.36 \%)$ \\
\hline P3: Axial Coma & $123.81[\mathrm{~nm}](16.95[\mathrm{~nm}])$ & $141.51^{\circ}(3.14 \%)$ \\
\hline A3: Three-fold astigmatism & $27.93[\mathrm{~nm}](8.49[\mathrm{~nm}])$ & $-8.72^{\circ}(7.21 \%)$ \\
\hline A4: Four-fold astigmatism & $0.305[\mu \mathrm{m}](0.15[\mu \mathrm{m}])$ & $-39.6^{\circ}(8.60 \%)$ \\
\hline Q4: Star aberration & $2.527[\mu \mathrm{m}](0.30[\mu \mathrm{m}])$ & $-13.98^{\circ}(2.01 \%)$ \\
\hline O4: $3^{\text {rd }}$ order spherical aberration & $1.597[\mu \mathrm{m}](0.82[\mu \mathrm{m}])$ & \\
\hline P5:4 ${ }^{\text {th }}$ order axial coma & $21.93[\mu \mathrm{m}](18.65[\mu \mathrm{m}])$ & $153.87^{\circ}(2.31 \%)$ \\
\hline R5:Three lobe aberration & $13.53[\mu \mathrm{m}](11.35[\mu \mathrm{m}])$ & $-7.78^{\circ}\left(18.92^{\circ} \%\right)$ \\
\hline A5:Five-fold astigmatism & $9.873[\mu \mathrm{m}](5.67[\mu \mathrm{m}])$ & $-26.43^{\circ}(8.57 \%)$ \\
\hline O6: $5^{\text {th }}$ order Spherical aberration & $-0.302[\mathrm{~mm}](0.76[\mathrm{~mm}])$ & \\
\hline A6: Six-fold astigmatism & $1.194[\mathrm{~mm}](0.15[\mathrm{~mm}])$ & $7.07^{\circ}(2.86 \%)$ \\
\hline
\end{tabular}

\section{Method Precision.}

We have measured defoci under constant electron-optical conditions to evaluate the precision of this method for comparison with a similar analysis using an amorphous sample. The value of the defocus was set at -65 $\mathrm{nm}$ and 100 Ronchigrams were recorded over a $7.5 \mathrm{~min}$ period on a Gatan OneView camera with $1 \mathrm{X}$ pixel binning (4096 $\times 4096$ pixel field of view) at a camera length of $80 \mathrm{~cm}$. An area of $1530 \times 1530$ pixels was selected with 1 pixel corresponding to $16.04 \mu \mathrm{rad}$. Subsequently, $16 \times 670 \times 670$ pixel auto-correlation patterns in a $4 \times 4$ array were calculated from within the experimental Ronchigram. The measured value of defoci are shown in Figure 7 (a). The linear term in the data of Figure 7 (a) gives a defocus $[\mathrm{nm}]=-64.36[\mathrm{~nm}]+0.057[\mathrm{~nm} / \mathrm{min}] \times \mathrm{t}[\mathrm{min}]$ which gives a specimen drift of $57 \mathrm{pm} / \mathrm{min}$ in the $z$ direction for the conditions used. To estimate the precision of the measurement, this linear term was subtracted from the data, as shown in Figure 7(b) giving a standard deviation in the measurements of $0.2408 \mathrm{~nm}$. In order to compare the precision of this method to that previously reported using the segmented Ronchigram auto-correlation matrix (SRAM) [9] data using an amorphous specimen was also acquired. For this latter dataset 100 Ronchigrams were also acquired at a camera length $40 \mathrm{~cm}$ for each defocus measurement using a $9 \times 9$ matrix of two images at defoci of $+70 \mathrm{~nm}$ and $-70 \mathrm{~nm}$. The linear specimen term was also subtracted from the raw data and the resultant measurements are shown in Figure 7(c) as a function of the number of measurements. The 
standard deviation calculated from Figure 7(c) is $1.374 \mathrm{~nm}$. Hence the precision of the measurement of defocus from the new method described using a two dimensional material is $5.7 \mathrm{X}$ better than the SRAM method using an amorphous film.

Figure 8 shows two-fold astigmatism measurements calculated from both the segmented Ronchigram using monolayer graphene and the SRAM method using an amorphous film. The mean value has been subtracted from both data sets to enable clear visualization of the precision in a single measurement. The standard deviation of the twofold astigmatism measurement from the segmentation of the Ronchigram using monolayer graphene (A2x $=0.25 \mathrm{~nm}$, $\mathrm{A} 2 \mathrm{y}=0.43 \mathrm{~nm})$ is less than half that as measured using an amorphous film (A2x $=1.00 \mathrm{~nm}, \mathrm{~A} 2 \mathrm{y}=0.88 \mathrm{~nm})$.

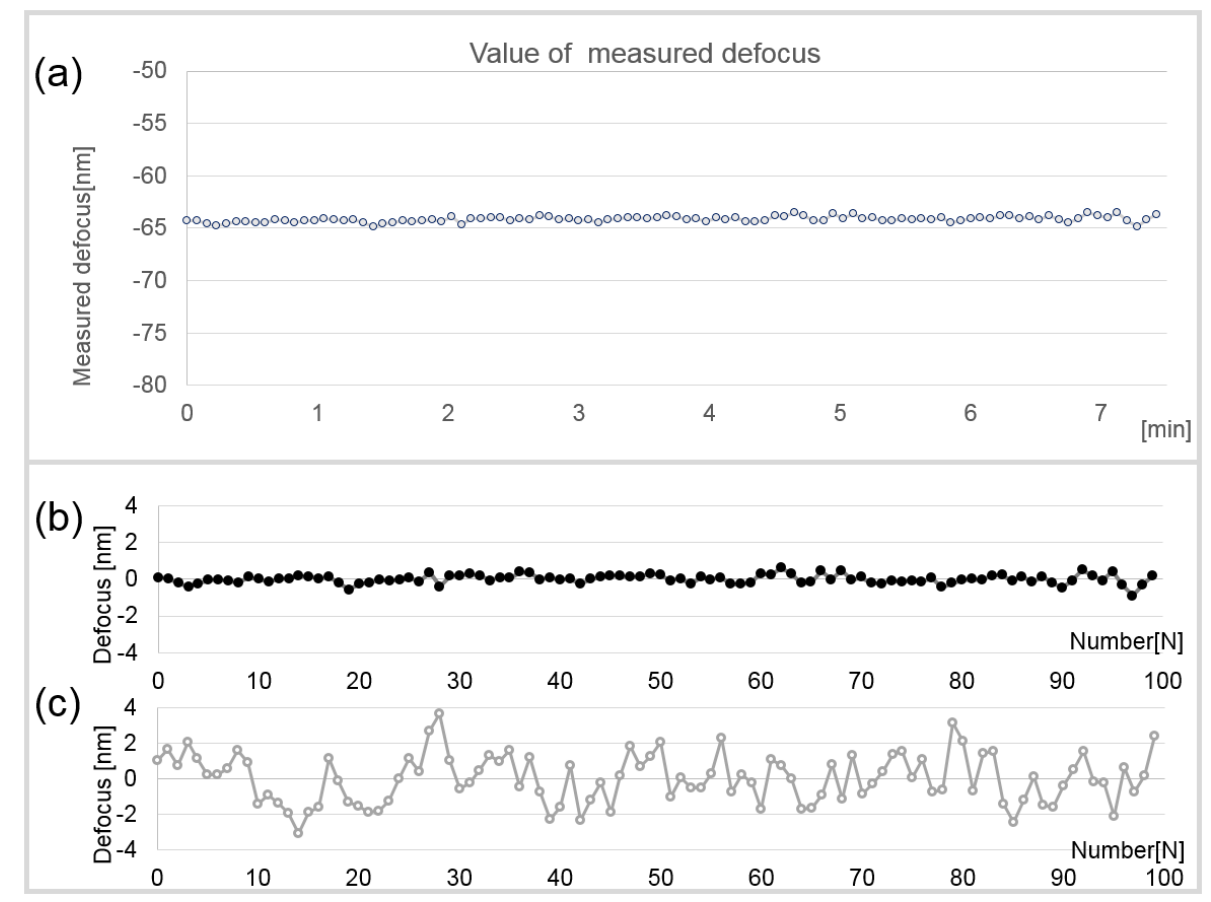

Figure 7. (a) Measurement of defocus as a function of time (minutes) for a constant objective defocus for 100 measurements using segmentation of Rochigrams recorded from monolayer graphene. (b) Measurement of defocus as a function of repeated measurement after subtracting the linear specimen drift term of $0.0569[\mathrm{~nm} / \mathrm{min}] \times \mathrm{t}[\mathrm{min}]$ from (a). (c) Measurement of defocus as a function of repeated measurement calculated from the SRAM using an amorphous film [9] after subtracting the linear specimen drift term of $0.519[\mathrm{~nm} / \mathrm{min}] \times \mathrm{t}[\mathrm{min}])$. 


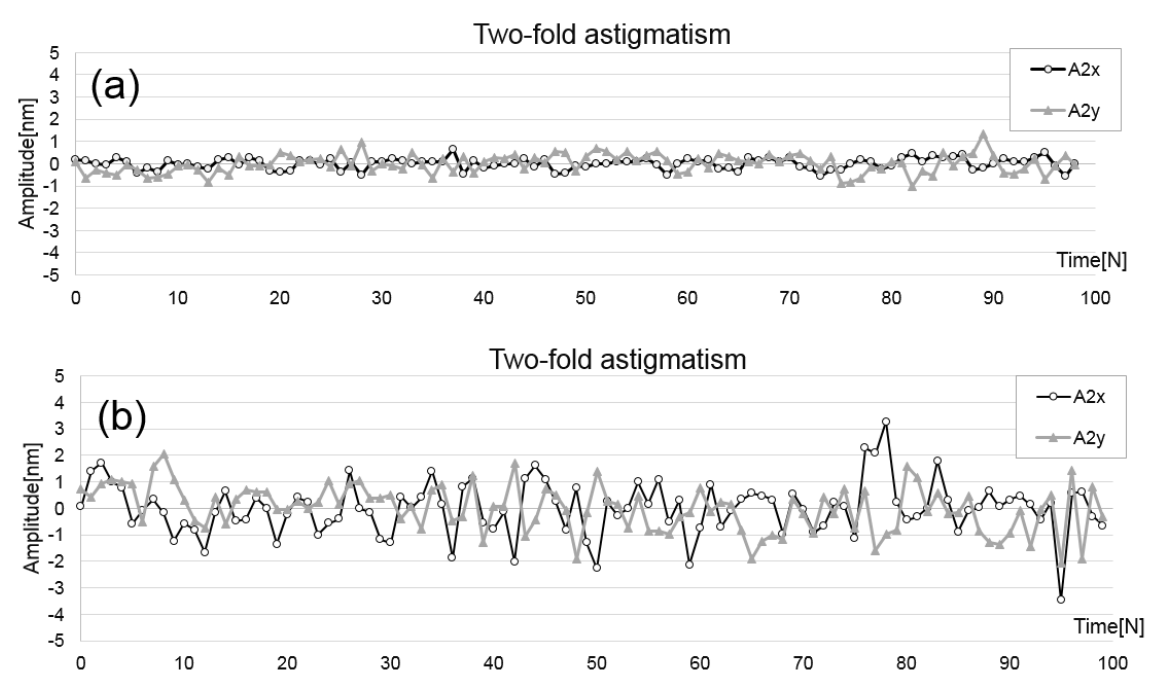

Figure 8. (a) Deviation from the mean two fold astigmatism in the $x$ (A2x) and $y$ (A2y) directions respectively measured using segmentation of the Rochigram from monolayer graphene. Standard deviations of the measurements in $\mathrm{A} 2 \mathrm{x}$ and $\mathrm{A} 2 \mathrm{y}$ are $0.25 \mathrm{~nm}$ and $0.43 \mathrm{~nm}$ respectively. (b) Deviation from the mean two-fold astigmatism measurement measured from the SRAM [9] using an amorphous film. The standard deviation of the measurements in $\mathrm{A} 2 \mathrm{x}$ and $\mathrm{A} 2 \mathrm{y}$ are $1.00 \mathrm{~nm}$ and $0.88 \mathrm{~nm}$ respectively.

\section{Aberration measurement using $\mathrm{MoS}_{2}$.}

This method described can easily be adapted to use other monolayer two-dimensional materials, such as $\mathrm{MoS}_{2}$ by simply changing the two-dimensional lattice parameter used for the analysis. To illustrate this a monolayer $\mathrm{MoS}_{2}$ sample (lattice coefficient $0.27 \mathrm{~nm}$ ) grown by chemical vapor deposition (CVD) [19] was used to measure the geometric aberration coefficients up to $3^{\text {rd }}$ order in the probe-forming system. A $3 \times 3$ array of $(120 \times 120$ pixel $)$ segmented sub-areas from a $200 \times 200$ pixel field of view was used in the calculations shown in Figure 9. Measured aberration coefficients are listed in Table 4. 

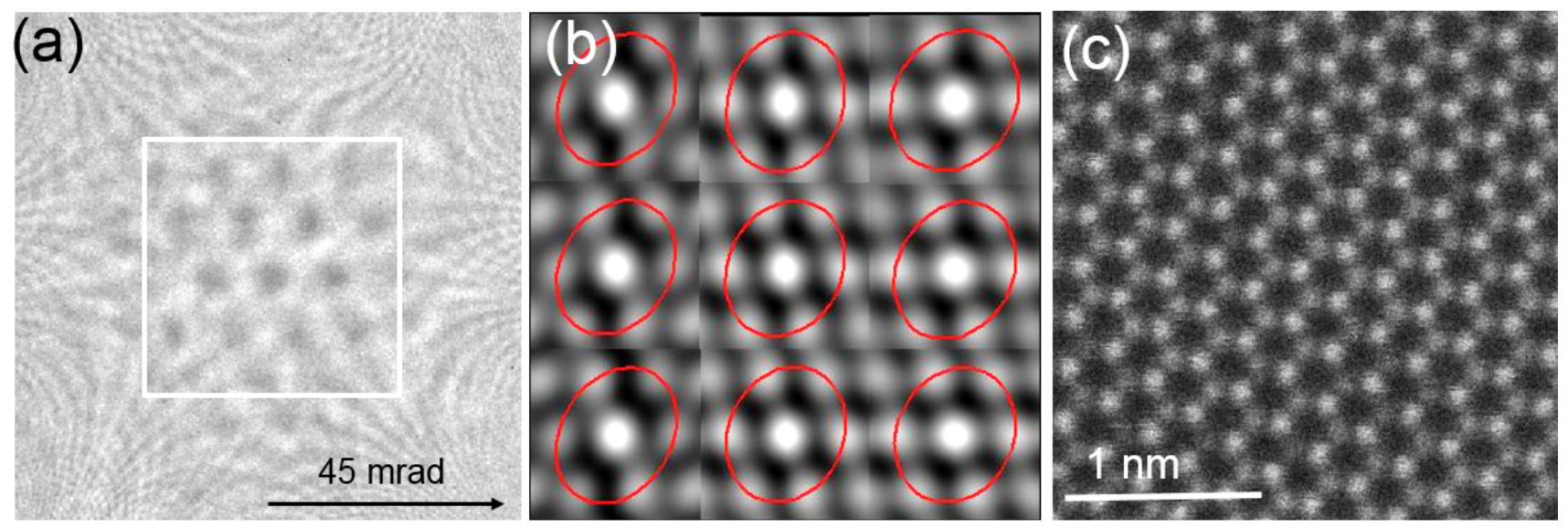

Figure 9. (a) Experimental Ronchigram $(361 \times 361$ pixels $)$ recorded from monolayer $\mathrm{MoS}_{2}$ with a STEM probe at a camera length of $20 \mathrm{~cm}$ at $80 \mathrm{kV}$. Auto-correlation patterns were calculated from the area indicated by the white square. (b) Local area auto-correlation patterns from an array of $3 \times 3$ sub-areas $(128 \times 128$ pixels $)$ and corresponding oval fits. Each auto-correlation pattern was reduced in size of $50 \%$ for display to avoid overlap with adjacent patterns. (c) LAADF image of monolayer $\mathrm{MoS}_{2}$ recorded at $80 \mathrm{kV}$ using the aberration conditions listed in Table A3. The STEM image acquisition parameters are same as those in Fig.6(c). The sampling time is $10 \mu \mathrm{s}$ pm/pixel for a sampling of $7.1 \mathrm{pm} / \mathrm{pixel}$.

Table 4: Measured aberration coefficients to fifth order of the probe forming system calculated from the measurements shown in Figure 9. Values in brackets correspond to errors in measurement values. The error in azimuth is described by $360^{\circ} /$ symmetry expressed as a percentage.

\begin{tabular}{|l|l|l|}
\hline Aberration & Amplitude & Azimuth \\
\hline O2: Defocus & $-21.35[\mathrm{~nm}](0.13[\mathrm{~nm}])$ & \\
\hline A2: Two-fold stigmatism & $1.952[\mathrm{~nm}](0.08[\mathrm{~nm}])$ & $64.49^{\circ}(0.81 \%)$ \\
\hline P3: Axial Coma & $54.55[\mathrm{~nm}](6.30[\mathrm{~nm}])$ & $-20.19^{\circ}(1.13 \%)$ \\
\hline A3: Three-fold astigmatism & $10.85[\mathrm{~nm}](1.41[\mathrm{~nm}])$ & $-3.491^{\circ}(10.03 \%)$ \\
\hline A4: Four-fold astigmatism & $1.478[\mu \mathrm{m}](0.46[\mu \mathrm{m}])$ & $-17.07^{\circ}(3.05 \%)$ \\
\hline Q4: Star aberration & $3.888[\mu \mathrm{m}](0.51[\mu \mathrm{m}])$ & $-28.14^{\circ}(3.55 \%)$ \\
\hline O4: $3^{\text {rd }}$ order spherical aberration & $0.998[\mu \mathrm{m}](0.37[\mu \mathrm{m}])$ & \\
\hline
\end{tabular}




\section{Measurement of chromatic aberration.}

As the presented method can measure the absolute value of the defocus in the probe-forming system to high precision, it can be applied to accurate measurement of chromatic aberration. A defocus spread $d f_{\mathrm{Cc}}$ due to chromatic aberration with an energy deviation of $d E$ is defined as $d f=-C c \cdot d E / E$.

A coefficient of chromatic aberration can be defined as,

$$
C c=-E \frac{d f_{C c}}{d E}
$$

where $E$ is the accelerating voltage.

The chromatic aberration coefficient after relativistic correction is given by $C c=C c^{*}(1+2 \varepsilon E) /(1+\varepsilon E)$, where $\varepsilon=0.9785 \times 10^{-6}[6]$ and $C_{c} *$ is a nonrelativistic chromatic aberration coefficient. $C_{c}$ can be derived from the slope of the defocus change with energy shift as given by Equation 9. Figure 10 shows Ronchigrams and corresponding auto-correlation functions recorded with increasing accelerating voltage from $80 \mathrm{kV}$ in increments, $d E=1 \mathrm{eV}$. The measured defocus value as a function of energy shift is shown in Figure 10 giving a value of $C c^{*}=$ $0.952 \mathrm{~mm}$ and $C c=1.02 \mathrm{~mm}$ at $80 \mathrm{kV}$ for the probe-forming system.
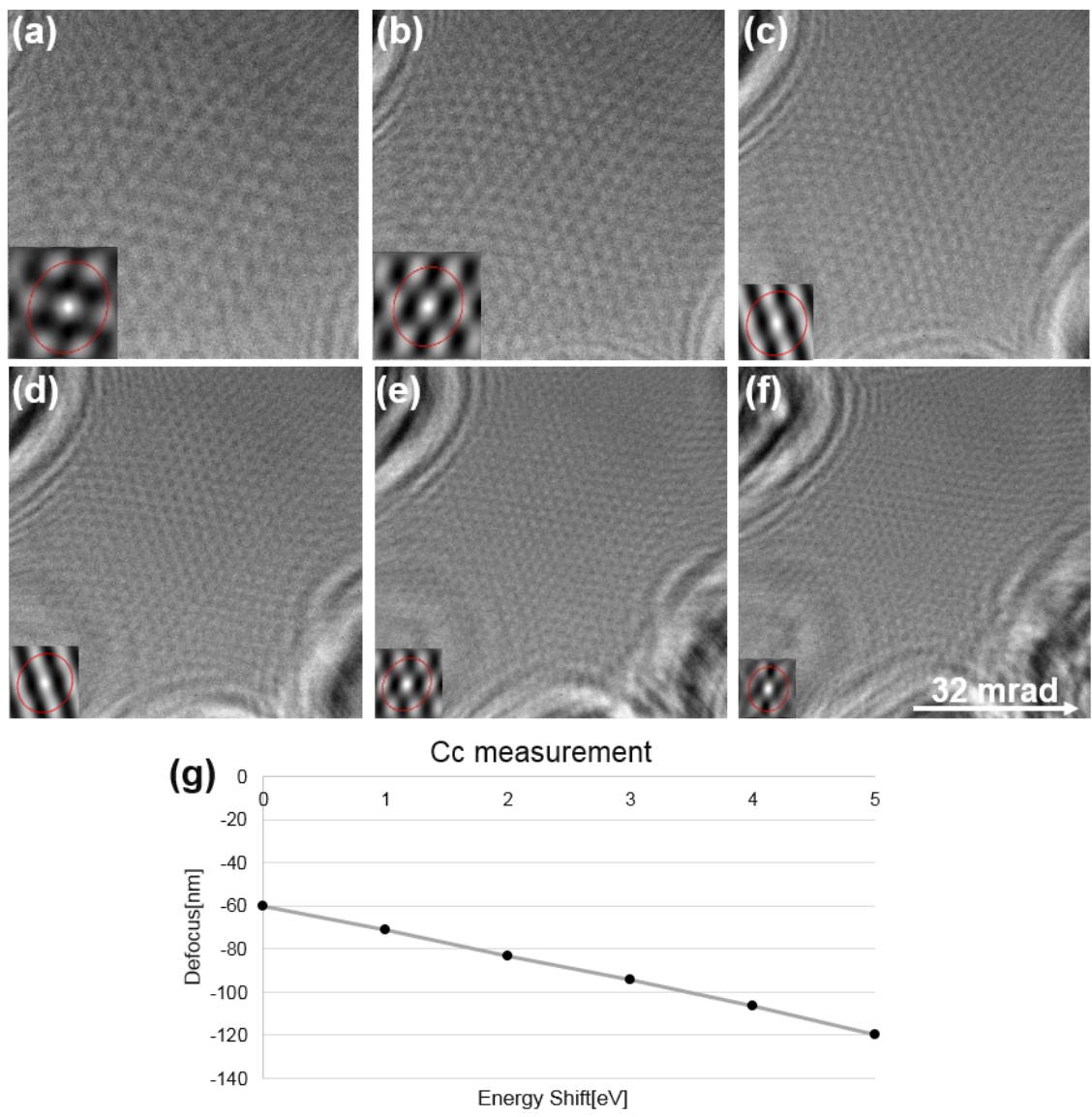

Figure 10. (a-f) Experimental Ronchigrams recorded from monolayer graphene with varying accelerating voltage. Insets show auto-correlation patterns used to measure defocus. (a) $d E=0 \mathrm{eV}$, (b) $d E=+1 \mathrm{eV}$, (c) $d E=+2 \mathrm{eV}$, (d) $d E$ $=+3 \mathrm{eV}$, (e) $d E=+4 \mathrm{eV}$ and (f) $d E=+5 \mathrm{eV}$. Ronchigrams were recorded at a camera length of $80 \mathrm{~cm}$ up to 32 mrad. (g) Value of defoci from 10 (a-f) as a function of energy shift. The slope is calculated to be $-11.9[\mathrm{~nm} / \mathrm{eV}]$ at $80 \mathrm{kV}$. 


\section{Conclusions}

We have described a method for accurate measurement of the axial geometric aberrations in a probe forming system up to $5^{\text {th }}$ order using analysis of a single Ronchigram measured from monolayer two-dimensional materials. Auto-correlation functions calculated from segmented angular areas have been used to estimate local distance and distortion in the two-dimensional lattice parameter measured from the Ronchigram. Substantially lower standard deviations in the measurement of defoci and two-fold astigmatism have been demonstrated compared to equivalent data recorded from an amorphous specimen measured using the previously reported SRAM. The validity of this method has also been demonstrated for measurements of higher order aberration coefficients and has been further applied to measure the coefficient of chromatic aberration for the probe-forming system.

\section{References}

[1] M. Haider, S. Uhlemann, E. Schwan, H. Rose, B. Kabius, and K. Urban, Nature 392 (1998) 768.

[2] O. L. Krivanek, M. F. Chisholm, V. Nicolosi, T. J. Pennycook, G. J. Corbin, N. Dellby, M. F. Murfitt, C. S. Own, Z. S. Szilagyi, M. P. Oxley, S. T. Pantelides, and S. J. Pennycook, Nature 464 (2010) 571.

[3] F. Hosokawa, H. Sawada, Y. Kondo, K. Takayanagi and K. Suenaga, Microscopy 62 (2013) 23.

[4] H. Sawada, T. Sasaki, F. Hosokawa, S. Yuasa, M. Terao, M. Kawazoe, T. Nakamichi, T. Kaneyama, Y. Kondo, K. Kimoto and K. Suenaga, J. Electron Microsc. 58 (2009) 341.

[5] M. Watanabe, Chapter 7 in Scanning Transmission Electron Microscopy: Imaging and Analysis, Eds. by Stephen J. Pennycook and Peter D. Nellist, Springer, NY, (2011) 291.

[6] M. Haider, S. Uhlemann and J. Zach, Ultramicroscopy 81 (2000) 163.

[7] N. Dellby, O. L. Krivanek, P. D. Nellist, P. E. Batson and A. R. Lupini, J. Electron Microsc. 50 (2001) 177.

[8] A. R. Lupini, P. Wang, P. D. Nellist, A. I. Kirkland and S. J, Pennycook, Ultramicroscopy 110 (2010) 891.

[9] H. Sawada, T. Sannomiya, F. Hosokawa, T. Nakamichi, T. Kaneyama, T. Tomita, Y. Kondo, T. Tanaka, Y.

Oshima, Y. Tanishiro and K. Takayanagi, Ultramicroscopy 108 (2008) 1467.

[10] A. R. Lupini and S. J.Pennycook, J. Electron Microsc. 57 (2008) 195.

[11] T. Yamazaki, Y. Kotaka, Y. Kikuchi and K. Watanabe, Ultramicroscopy 106 (2006) 153.

[12] K. Kuramochi, T. Yamazaki, Y. Kotaka, Y. Kikuchi, I. Hashimoto and K. Watanabe, Ultramicroscopy 108 (2008) 339.

[13] Q. M. Ramasse and A. L. Bleloch Ultramicroscopy 106 (2005) 37.

[14] H. Yang, R.N. Rutte, L. Jones, M. Simson, R. Sagawa, H. Ryll, M. Huth, T.J. Pennycook, H. Soltau, Y. Kondo, B.D. Davis and P.D. Nellist, Nature Communications 7 (2016) 12532

[15] Graphene samples from Graphenea Inc. (https://www.graphenea.com)

[16] V. Ronchi, Appl. Opt. 3 (1964) 437.

[17] J. M. Cowley, Ultramicroscopy 4 (1979) 435.

[18] J. M. Cowley, J. Electron Microscopy Technique 3 (1986) 25.

[19] S. Wang, M. Pacios, H. Bhaskaran and J. H. Warner, Nanotechnology 27 (2016) 85604. 


\section{Appendix 1: Geometric Aberrations.}

The complex geometric aberration coefficients are listed in Table A1. The complex angle $\omega=\alpha \exp (\mathrm{i} \theta)=\omega_{u}+\mathrm{i} \omega_{v}=\lambda(u+\mathrm{i} v)$ is defined by a convergence angle $\alpha$, with an azimuth $\theta$, where $\lambda$ is the wavelength of the incident electrons.

Table A1. Definition of geometric aberrations and aberration coefficients.

\begin{tabular}{|c|c|c|c|}
\hline Axial aberration & Notation & Coefficient & Geometric aberration \\
\hline Defocus & $\boldsymbol{O}_{\mathbf{2}}$ & $O_{2}$ & $O_{2} \boldsymbol{\omega}$ \\
\hline Two-fold astigmatism & $\boldsymbol{A}_{\mathbf{2}}$ & $a_{2} \exp \left(2 \mathrm{i} \theta_{a 2}\right)$ & $A_{2} \overline{\boldsymbol{\omega}}$ \\
\hline Axial coma & $\boldsymbol{P}_{\mathbf{3}}$ & $p_{3} \exp \left(\mathrm{i} \theta_{p 3}\right)$ & $1 / 3 \cdot \overline{P_{3}} \boldsymbol{\omega}^{2}+2 / 3 \cdot P_{3} \overline{\boldsymbol{\omega}} \boldsymbol{\omega}$ \\
\hline Three-fold astigmatism & $\boldsymbol{A}_{\mathbf{3}}$ & $a_{3} \exp \left(3 \mathrm{i} \theta_{a 3}\right)$ & $A_{3} \overline{\boldsymbol{\omega}}^{2}$ \\
\hline Third order spherical aberration & $\boldsymbol{O}_{\mathbf{4}}$ & $O_{4}$ & $O_{4} \overline{\boldsymbol{\omega}} \boldsymbol{\omega}^{2}$ \\
\hline Star aberration & $\boldsymbol{Q}_{\mathbf{4}}$ & $q_{4} \exp \left(2 \mathrm{i} \theta_{q 4}\right)$ & $1 / 4 \cdot \overline{Q_{4}} \boldsymbol{\omega}^{3}+3 / 4 \cdot Q_{4} \overline{\boldsymbol{\omega}}^{2} \boldsymbol{\omega}$ \\
\hline Four-fold astigmatism & $\boldsymbol{A}_{\mathbf{4}}$ & $a_{4} \exp \left(4 \mathrm{i} \theta_{a 4}\right)$ & $A_{4} \overline{\boldsymbol{\omega}}^{3}$ \\
\hline Fourth order axial coma & $\boldsymbol{P}_{\mathbf{5}}$ & $p_{5} \exp \left(\mathrm{i} \theta_{p 5}\right)$ & $2 / 5 \cdot \bar{P}_{5} \overline{\boldsymbol{\omega}}^{3}+3 / 5 \cdot P_{5} \overline{\boldsymbol{\omega}}^{2} \boldsymbol{\omega}^{2}$ \\
\hline Three lobe aberration & $\boldsymbol{R}_{\mathbf{5}}$ & $r_{5} \exp \left(3 \mathrm{i} \theta_{r 5}\right)$ & $1 / 5 \cdot \bar{R}_{5} \boldsymbol{\omega}^{4}+4 / 5 \cdot R_{5} \overline{\boldsymbol{\omega}}^{3} \boldsymbol{\omega}$ \\
\hline Five-fold astigmatism & $\boldsymbol{A}_{\mathbf{5}}$ & $a_{5} \exp \left(5 \mathrm{i} \theta_{a 5}\right)$ & $A_{5} \overline{\boldsymbol{\omega}}^{4}$ \\
\hline Fifth order spherical aberration & $\boldsymbol{O}_{\mathbf{6}}$ & $o_{6}$ & $O_{6} \overline{\boldsymbol{\omega}}^{2} \boldsymbol{\omega}^{3}$ \\
\hline Six-fold astigmatism & $\boldsymbol{A}_{\mathbf{6}}$ & $a_{6} \exp \left(6 \mathrm{i} \theta_{a 6}\right)$ & $A_{6} \overline{\boldsymbol{\omega}}^{5}$ \\
\hline
\end{tabular}


Table A2. Factors $A, B$, and $C$ [9].

\begin{tabular}{|c|c|c|c|}
\hline & $A$ & $B$ & $C$ \\
\hline $\mathrm{O}_{2}$ & $\mathrm{O}_{2}$ & 0 & $\mathrm{O}_{2}$ \\
\hline$A_{2}$ & $a_{2} \cos 2\left(\theta_{a 2}\right)$ & $a_{2} \sin 2\left(\theta_{a 2}\right)$ & $-a_{2} \cos 2\left(\theta_{a 2}\right)$ \\
\hline$P_{3}$ & $\begin{array}{l}4 / 3 p_{3} \alpha \cos \left(\theta-\theta_{p 3}\right) \\
+2 / 3 p_{3} \alpha \cos \left(\theta+\theta_{p 3}\right)\end{array}$ & $2 / 3 \cdot p_{3} \alpha \sin \left(\theta+\theta_{p 3}\right)$ & $\begin{array}{l}4 / 3 p_{3} \alpha \cos \left(\theta-\theta_{p 3}\right) \\
-2 / 3 p_{3} \alpha \cos \left(\theta+\theta_{p 3}\right)\end{array}$ \\
\hline$A_{3}$ & $2 a_{3} \alpha \cos \left(\theta-3 \theta_{a 3}\right)$ & $-2 a_{3} \alpha \sin \left(\theta-3 \theta_{a 3}\right)$ & $-2 a_{3} \alpha \cos \left(\theta-3 \theta_{a 3}\right)$ \\
\hline $\mathrm{O}_{4}$ & $2 o_{4} \alpha^{2}+o_{4} \alpha^{2} \cos 2 \theta$ & $o_{4} \alpha^{2} \sin 2 \theta$ & $2 o_{4} \alpha^{2}-o_{4} \alpha^{2} \cos 2 \theta$ \\
\hline$Q_{4}$ & $3 / 2 \cdot q_{4} \alpha^{2} \cos \left(\theta_{q^{4}}-2 \theta\right)+3 / 2 \cdot q_{4} \alpha^{2}$ & 0 & $3 / 2 \cdot q_{4} \alpha^{2} \cos \left(\theta_{q^{4}}-2 \theta\right)-3 / 2 \cdot q_{4} \alpha^{2}$ \\
\hline$A_{4}$ & $3 a_{4} \alpha^{2} \cos \left(2 \theta-4 \theta_{a 4}\right)$ & $-3 a_{4} \alpha^{2} \sin \left(2 \theta-4 \theta_{a 4}\right)$ & $-3 a_{4} \alpha^{2} \cos \left(2 \theta-4 \theta_{a 4}\right)$ \\
\hline$P_{5}$ & $\begin{array}{l}12 / 5 p_{5} \alpha^{3} \cos \left(\theta-\theta_{p 5}\right) \\
+6 / 5 p_{5} \alpha^{3} \cos \left(\theta+\theta_{p 5}\right) \\
+2 / 5 p_{5} \alpha^{3} \cos \left(3 \theta-\theta_{p 5}\right)\end{array}$ & $\begin{array}{l}6 / 5 p_{5} \alpha^{3} \sin \left(\theta+\theta_{p 5}\right) \\
+2 / 5 p_{5} \alpha^{3} \sin \left(3 \theta-\theta_{p 5}\right)\end{array}$ & $\begin{array}{l}12 / 5 p_{5} \alpha^{3} \cos \left(\theta-\theta_{p 5}\right) \\
-6 / 5 p_{5} \alpha^{3} \cos \left(\theta+\theta_{p 5}\right) \\
-2 / 5 p_{5} \alpha^{3} \cos \left(3 \theta-\theta_{p 5}\right)\end{array}$ \\
\hline$R_{5}$ & $\begin{array}{l}8 / 5 r_{5} \alpha^{3} \cos \left(3 \theta-\theta_{r 5}\right) \\
+12 / 5 r_{5} \alpha^{3} \cos \left(\theta-\theta_{r 5}\right)\end{array}$ & $-12 / 5 r_{5} \alpha^{3} \sin \left(\theta-\theta_{r 5}\right)$ & $\begin{array}{l}8 / 5 r_{5} \alpha^{3} \cos \left(3 \theta-\theta_{r 5}\right) \\
-12 / 5 r_{5} \alpha^{3} \cos \left(\theta-\theta_{r 5}\right)\end{array}$ \\
\hline$A_{5}$ & $4 a_{5} \alpha^{3} \cos \left(3 \theta-5 \theta_{a 5}\right)$ & $-4 a_{5} \alpha^{3} \sin \left(3 \theta-5 \theta_{a 5}\right)$ & $-4 a_{5} \alpha^{3} \cos \left(3 \theta-5 \theta_{a 5}\right)$ \\
\hline$O_{6}$ & $3 o_{6} \alpha^{4}+2 \alpha^{4} o_{6} \cos 2 \theta$ & $2 \alpha^{4} o_{6} \sin 2 \theta$ & $3 o_{6} \alpha^{4}-2 \alpha^{4} o_{6} \cos 2 \theta$ \\
\hline$A_{6}$ & $5 a_{6} \alpha^{4} \cos \left(4 \theta-6 \theta_{a 6}\right)$ & $-5 a_{6} \alpha^{4} \sin \left(4 \theta-6 \theta_{a 6}\right)$ & $-5 a_{6} \alpha^{4} \cos \left(4 \theta-6 \theta_{a 6}\right)$ \\
\hline
\end{tabular}

